\title{
Q-CURVATURE FLOW WITH INDEFINITE NONLINEARITY
}

\author{
LI MA
}

\begin{abstract}
In this note, we study Q-curvature flow on $S^{4}$ with indefinite nonlinearity. Our result is that the prescribed Q-curvature problem on $S^{4}$ has a solution provided the prescribed Q-curvature $f$ has its positive part, which possesses non-degenerate critical points such that $\Delta_{S^{4}} f \neq 0$ at the saddle points and an extra condition such as a nontrivial degree counting condition.
\end{abstract}

Mathematics Subject Classification 2000: 53Cxx,35Jxx

Keywords: Q-curvature flow, indefinite nonlinearity, blow-up, conformal class

\section{INTRODUCTION}

Following the works of A.Chang-P.Yang [4], M.Brendle [3], Malchiodi and M.Struwe [6], we study a heat flow method to the prescribed Q-curvature problem on $S^{4}$. Given the Riemannian metric $g$ in the conformal class of standard metric $c$ on $S^{4}$ with Q-curvature $Q_{g}$. Then it is the well-known that

$$
Q_{g}=-\frac{1}{12}\left(\Delta_{g} R_{g}-R_{g}^{2}+3|R c(g)|^{2}\right):=Q,
$$

where $R_{g}, R c(g), \Delta_{g}$ are the scalar curvature, Ricci curvature tensor, the Laplacian operator of the metric $g$ respectively.

Recall the Chern-Gaussian-Bonnet formula on $S^{4}$ is

$$
\int_{S^{4}} Q_{g} d v_{g}=8 \pi^{2}
$$

Hence,we know that $Q_{g}$ has to be positive somewhere. This gives a necessary condition for the prescribed Q-curvature problem on $S^{4}$. Assuming the prescribed curvature function $f$ being positive on $S^{4}$, the heat flow for the Q-curvature problem is a family of metrics of the form $g=e^{2 u(x, t)} c$ satisfying

$$
u_{t}=\alpha f-Q, \quad x \in S^{4}, \quad t>0,
$$

where $u: S^{4} \times(0, T) \rightarrow R$, and $\alpha=\alpha(t)$ is defined by

$$
\alpha \int_{S^{4}} f d v_{g}=8 \pi^{2}
$$

Date: July 12th, 2008.

* The research is partially supported by the National Natural Science Foundation of China 10631020 and SRFDP 20060003002 . 
Here $d v_{g}$ is the area element with respect to the metric $g$. It is easy to see that

$$
\alpha_{t} \int_{S^{4}} f d v_{g}=2 \alpha \int_{S^{4}}(Q-\alpha f) f d v_{g} .
$$

Malchiodi and M.Struwe [6] can show that the flow exists globally, furthermore, the flow converges at infinity provided $f$ possesses non-degenerate critical points such that $\Delta_{S^{4}} f \neq 0$ at the saddle points with the condition

$$
\sum_{\left\{p: \nabla f(p)=0 ; \Delta_{S^{4}} f(p)<0\right\}}(-1)^{i n d(f, p)} \neq 0 .
$$

Here $\Delta_{S^{4}}:=\Delta$ is the Analyst's Laplacian on the standard 4-sphere $\left(S^{4}, c\right)$. Recall that $\int_{S^{4}} d v_{c}=\frac{8}{3} \pi^{2}$. The purpose of this paper is to relax their assumption by allowing the function $f$ to have sign-changing or to have zeros.

Since we have

$$
Q=\frac{1}{2} e^{-4 u}\left(\Delta^{2} u-\operatorname{div}\left(\left(\frac{2}{3} R(c) c-2 R c(c)\right) d u\right)+6\right),
$$

the equation (11) define a nonlinear parabolic equation for $u$, and the flow exists at least locally for any initial data $\left.u\right|_{t=0}=u_{0}$. Clearly, we have

$$
\partial_{t} \int_{S^{4}} d v_{g}=2 \int S^{4} u_{t} d v_{g}=0 .
$$

We shall assume that the initial data $u_{0}$ satisfies the condition

$$
\int f e^{4 u} d v_{c}>0
$$

We shall show that this property is preserved along the flow. It is easy to compute that

$$
Q_{t}=-4 u_{t} Q-\frac{1}{2} P u_{t}=4 Q(Q-\alpha f)+P(\alpha f-Q),
$$

where $P=P_{g}=e^{-4 u} P_{c}$ and $P_{c}$ is the Paneitz operator in the metric $c$ on $S^{4}$ [4]. Using (44), we can compute the growth rate of the Calabi-type energy $\int_{S^{4}}|Q-\alpha f|^{2} d v_{g}$.

Our main result is following

Theorem 1. Let $f$ be a positive somewhere, smooth function on $S^{4}$ with only non-degenerate critical points on the its positive part $f_{+}$with its Morse index ind $\left(f_{+}, p\right)$. Suppose that at each critical point $p$ of $f_{+}$, we have $\Delta f \neq 0$. Let $m_{i}$ be the number of critical points with $f(p)>0, \Delta_{S^{4}} f(p)<0$ and ind $(f, p)=4-i$. Suppose that there is no solutions with coefficients $k_{i} \geq 0$ to the system of equations

$$
m_{0}=1+k_{0}, m_{i}=k_{i-1}+k_{i}, 1 \leq i \leq 4, k_{4}=0 .
$$

Then $f$ is the $Q$ curvature of the conformal metric $g=e^{2 u} c$ on $S^{4}$. 
Note that this result is an extension of the famous result of MalchiodiStruwe [6] where only positive $f$ has been considered. A similar result for Curvature flow to Nirenberg problem on $S^{2}$ has been obtained in [8]. See also J.Wei and X.Xu's work [9].

For simplifying notations, we shall use the conventions that $d c=\frac{d v_{c}}{\frac{8}{3} \pi^{2}}$ and $\bar{u}=\bar{u}(t)$ defined by

$$
\int_{S^{4}}(u-\bar{u}) d v_{c}=0
$$

\section{BASIC PROPERTIES OF THE FLOW}

Recall the following result of Beckner [2] that

$$
\int_{S^{4}}\left(|\Delta u|^{2}+2|\nabla u|^{2}+12 u\right) d c \geq \log \left(\int_{S^{4}} e^{4 u} d c\right)=0
$$

where $|\nabla u|^{2}$ is the norm of the gradient of the function $u$ with respect to the standard metric $c$. Here we have used the fact that $\int_{S^{4}} e^{4 u} d c=1$ along the flow (11).

We show that this condition is preserved along the flow (11). In fact, letting

$$
E(u)=\int_{S^{4}}\left(u P u+4 Q_{c} u\right) d c=\int_{S^{4}}\left(|\Delta u|_{c}^{2}+2|\nabla u|_{c}^{2}+12 u\right) d c
$$

be the Liouville energy of $u$ and letting

$$
E_{f}(u)=E(u)-3 \log \left(\int_{S^{4}} f e^{4 u} d c\right)
$$

be the energy function for the flow (1), we then compute that

$$
\partial_{t} E_{f}(u)=-\frac{3}{2 \pi^{2}} \int_{S^{4}}|\alpha f-Q|^{2} d v_{g} \leq 0 .
$$

One may see Lemma 2.1 in [6] for a proof. Hence

$$
E_{f}(u(t)) \leq E_{f}\left(u_{0}\right), \quad t>0 .
$$

After using the inequality (5) we have

$$
\log \left(1 / \int_{S^{4}} f e^{4 u} d c\right) \leq E_{f}\left(u_{0}\right),
$$

which implies that $\int_{S^{4}} f e^{4 u} d v_{c}>0$ and

$$
e^{E_{f}\left(u_{0}\right)} \int_{S^{4}} e^{4 u} d c \leq \int_{S^{4}} f e^{4 u} d c .
$$

Note also that $\int_{S^{4}} f e^{4 u} d c=1 / \alpha(t)$. Hence,

$$
\alpha(t) \leq \frac{1}{e^{E_{f}\left(u_{0}\right)}} .
$$


Using the definition of $\alpha(t)$ we have

$$
\alpha(t) \geq \frac{1}{\max _{S^{4}} f} .
$$

We then conclude that $\alpha(t)$ is uniformly bounded along the flow, i.e.,

$$
\frac{1}{\max _{S^{4}} f} \leq \alpha(t) \leq \frac{1}{e^{E_{f}\left(u_{0}\right)}} .
$$

We shall use this inequality to replace (26) in [6] in the study of the normalized flow, which will be defined soon following the work of Machiodi and M.Struwe [6]. If we have a global flow, then using (6) we have

$$
2 \int_{0}^{\infty} d t \int_{S^{4}}|\alpha f-Q|^{2} d v_{g} \leq 4 \pi\left(E_{f}\left(u_{0}\right)+\log \max _{S^{4}} f\right) .
$$

Hence we have a suitable sequence $t_{l} \rightarrow \infty$ with associated metrics $g_{l}=$ $g\left(t_{l}\right)$ and $\alpha\left(t_{l}\right) \rightarrow \alpha>0$, and letting $Q_{l}=Q\left(g_{l}\right)$ be the Q-curvature of the metric $g_{l}$, such that

$$
\int_{S^{4}}\left|Q_{l}-\alpha f\right|^{2} \rightarrow 0, \quad\left(t_{l} \rightarrow \infty\right)
$$

Therefore, once we have a limiting metric $g_{\infty}$ of the sequence of the metrics $g_{l}$, it follows that $Q\left(g_{\infty}\right)=\alpha f$. After a re-scaling, we see that $f$ is the Gaussian curvature of the metric $\beta g_{\infty}$ for some $\beta>0$, which implies our Theorem 1,

\section{Normalized flow AND the proof of Theorem 1}

We now introduce a normalized flow. For the given flow $g(t)=e^{2 u(t)} c$ on $S^{4}$, there exists a family of conformal diffeomorphisms $\phi=\phi(t): S^{4} \rightarrow S^{4}$, which depends smoothly on the time variable $t$, such that for the metrics $h=\phi^{*} g$, we have

$$
\int_{S^{4}} x d v_{h}=0, \text { for all } t \geq 0 .
$$

Here $x=\left(x^{1}, x^{2}, x^{3}, x^{4}, x^{5}\right) \in S^{4} \subset R^{5}$ is a position vector of the standard 4-sphere. Let

$$
v=u \circ \phi+\frac{1}{4} \log (\operatorname{det}(d \phi)) .
$$

Then we have $h=e^{2 v} c$. Using the conformal invariance of the Liouville energy [4, we have

$$
E(v)=E(u),
$$

and furthermore,

$$
\operatorname{Vol}\left(S^{4}, h\right)=\operatorname{Vol}\left(S^{4}, g\right)=\frac{8}{3} \pi^{2}, \text { for all } t \geq 0 .
$$

Assume $u(t)$ satisfies (11) and (2). Then we have the uniform energy bounds

$$
0 \leq E(v) \leq E(u)=E_{f}(u)+\log \left(\int_{S^{4}} f e^{4 u} d c\right) \leq E_{f}\left(u_{0}\right)+\log \left(\max _{S^{4}} f\right) .
$$


Using Jensen's inequality we have

$$
2 \bar{v}:=\int_{S^{4}} 2 v d c \leq \log \left(\int_{S^{4}} e^{4 v} d c\right)=0 .
$$

Using this we can obtain the uniform $H^{1}$ norm bounds of $v$ for all $t \geq 0$ that

$$
\sup _{t}|v(t)|_{H^{1}\left(S^{2}\right)} \leq C .
$$

See the proof of Lemma 3.2 in [6]. Using the Aubin-Moser-Trudinger inequality [1] we further have

$$
4 \sup _{\{0 \leq t<T\}} \int_{S^{4}}|u(t)| d c \leq \sup _{t} \int_{S^{4}} e^{4|u(t)|} d c \leq C<\infty .
$$

Note that

$$
v_{t}=u_{t} \circ \phi+\frac{1}{4} e^{-4 v} \operatorname{div}_{S^{4}}\left(\xi e^{4 v}\right)
$$

where $\xi=(d \phi)^{-1} \phi_{t}$ is the vector field on $S^{2}$ generating the flow $(\phi(t))$, $t \geq 0$, as in [6], formula (17), with the uniform bound

$$
|\xi|_{L^{\infty}\left(S^{4}\right)}^{2} \leq C \int_{S^{4}}|\alpha f-K|^{2} d v_{g}
$$

With the help of this bound, we can show (see Lemma 3.3 in [6]) that for any $T>0$, it holds

$$
\sup _{0 \leq t<T} \int_{S^{2}} e^{4|u(t)|} d c<+\infty .
$$

Following the method of Malchiodi and M.Struwe [6] (see also Lemma 3.4 in [5]) and using the bound (8) and the growth rate of $\alpha$, we can show that

$$
\int_{S^{4}}|\alpha f-Q|^{2} d v_{g} \rightarrow 0
$$

as $t \rightarrow \infty$. Once getting this curvature decay estimate, we can come to consider the concentration behavior of the metrics $g(t)$. Following [5], we show that

Lemma 2. Let $\left(u_{l}\right)$ be a sequence of smooth functions on $S^{4}$ with associated metrics $g_{l}=e^{2 u_{l}} c$ with $\operatorname{vol}\left(S^{4}, g_{l}\right)=\frac{8}{3} \pi^{2}, l=1,2, \ldots$ as constructed above. Suppose that there is a smooth function $Q_{\infty}$, which is positive somewhere in $S^{4}$ such that

$$
\left|Q\left(g_{l}\right)-Q_{\infty}\right|_{L^{2}\left(S^{4}, g_{l}\right)} \rightarrow 0
$$

as $l \rightarrow \infty$. Let $h_{l}=\phi_{l}^{*} g_{l}=e^{2 v_{l}} c$ be defined as before. Then we have either

1) for a subsequence $l \rightarrow \infty$ we have $u_{l} \rightarrow u_{\infty}$ in $H^{4}\left(S^{4}, c\right)$, where $g_{\infty}=$ $e^{2 u_{\infty}}$ c has $Q$ - curvature $Q_{\infty}$, or

2) there exists a subsequence, still denoted by $\left(u_{l}\right)$ and a point $q \in S^{4}$ with $Q_{\infty}(q)>0$, such that the metrics $g_{l}$ has a measure concentration that

$$
d v_{g_{l}} \rightarrow \frac{8}{3} \pi^{2} \delta_{q}
$$


weakly in the sense of measures, while $h_{l} \rightarrow c$ in $H^{4}\left(S^{4}, c\right)$ and in particular, $Q\left(h_{l}\right) \rightarrow 3$ in $L^{2}\left(S^{4}\right)$. Moreover, in the latter case the conformal diffeomorphisms $\phi_{l}$ weakly converges in $H^{2}\left(S^{4}\right)$ to the constant map $\phi_{\infty}=q$.

Proof. The case 1) can be proved as Lemma 3.6 in [6]. So we need only to prove the case 2). As in [6], we choose $q_{l} \in S^{4}$ and radii $r_{l}>0$ such that

$$
\sup _{q \in S^{4}} \int_{B\left(q, r_{l}\right)}\left|K\left(g_{l}\right)\right| d v_{g_{l}} \leq \int_{B\left(q_{l}, r_{l}\right)}\left|K\left(g_{l}\right)\right| d v_{g_{l}}=2 \pi^{2},
$$

where $B\left(q, r_{l}\right)$ is the geodesic ball in $\left(S^{4}, g_{l}\right)$. Then we have $r_{l} \rightarrow 0$ and we may assume that $q_{l} \rightarrow q$ as $l \rightarrow \infty$. For each $l$, we introduce $\phi_{l}$ as in Lemma 3.6 in [6] so that the functions

$$
\hat{u}_{l}=u_{l} \circ \phi_{l}+\frac{1}{4} \log \left(\operatorname{det}\left(d \phi_{l}\right)\right)
$$

satisfy the conformal Q-curvature equation

$$
-P_{R^{4}} \hat{u}_{l}=2 \hat{Q}_{l} e^{4 \hat{u}_{l}}, \text { on } R^{4},
$$

where $\hat{Q}_{l}=Q\left(g_{l}\right) \circ \phi$ and $P_{R^{4}}$ is the Paneitz operator of the standard Euclidean metric $g_{R^{4}}$. Note that for $\hat{g}_{l}=\phi^{*} g_{l}=e^{2 \hat{u}_{l}} g_{R^{4}}$, we have

$$
\operatorname{Vol}\left(R^{4}, \hat{g}_{l}\right)=\operatorname{Vol}\left(S^{4}, g_{l}\right)=\frac{8}{3} \pi^{2} \text {. }
$$

Arguing as in [6], we can conclude a convergent subsequence $\hat{u}_{l} \rightarrow \hat{u}_{\infty}$ in $H_{l o c}^{4}\left(R^{4}\right)$ where $\hat{u}_{\infty}$ satisfies the Liouville type equation

$$
-\Delta_{R^{4}}^{2} \hat{u}_{\infty}=\hat{Q}_{\infty}(q) e^{4 \hat{u}_{\infty}} \text {, on } R^{4},
$$

with

$$
\int_{R^{4}} K_{\infty}(q) e^{4 \hat{u}_{\infty}} d z \leq \frac{8}{3} \pi^{2}
$$

We only need to exclude the case when $Q_{\infty}(q) \leq 0$. Just note that by (7) we have

$$
\log \left(1 / \int_{R^{4}} f \circ \phi_{l} e^{4 \hat{u}_{l}}\right) \leq E_{f}\left(u_{0}\right) .
$$

Hence, sending $l \rightarrow \infty$, we always have $f \circ \phi_{l} \rightarrow f \circ \phi(q)>0$ uniformly on any compact domains of $R^{4}$.

The remaining part is the same as in the proof of Lemma 3.6 in [6]. We confer to [6] for the full proof.

We remark that some other argument can also exclude the case $Q_{\infty}(q)<$ 0 . It can not occur since there is no such a solution on the whole space $R^{4}$ (see also the argument in [7]). If $Q_{\infty}(q)=0$, then $\Delta_{R^{4}} \hat{u}:=\Delta_{R^{4}} \hat{u}_{\infty}$ is a harmonic function in $R^{4}$. Let $\bar{u}(r)$ be the average of $u$ on the circle $\partial B_{r}(0) \subset R^{4}$. Then we have

$$
\Delta_{R^{4}}^{2} \bar{u}=0 .
$$


Hence $\Delta_{R^{4}} \bar{u}=A+B r^{-2}$ for some constants $A$ and $B$, where $r=|x|$. Since $\Delta_{R^{4}} \bar{u}$ is a continuous function on $[0, \infty)$, we have $\Delta_{R^{4}} \bar{u}=A$, which gives us that

$$
\bar{u}=A+B r^{2}+C r^{-2}
$$

for some constants $A, B$, and $C$. Again, using $\bar{u}$ is regular, we have $C=0$ and $\bar{u}=A+B r^{2}$ with $B<0$. However, it seems hard to exclude this case without the use of the fact (7).

With this understanding, we can do the same finite-dimensional dynamics analysis as in section 5 in [6]. Then arguing as in section 5 in [6] we can prove Theorem 1. By now the argument is well-known, so we omit the detail and refer to [6] for full discussion.

\section{REFERENCES}

[1] Aubin, Thierry: Some nonlinear problems in Riemannian geometry (Springer Monographs in Mathematics). Springer, Berlin 1998 MR1636569 (99i:58001)

[2] W.Beckner, Sharp Sobolev inequalities on the sphere and the Moser-Trudinger inequality, Ann. Math., 138(1993)213-242.

[3] , S.Brendle, Global existence and convergence for a higher order flow in conformal geometry, Ann. math., 158(2003)323-343.

[4] S.Y.A.Chang, P.Yang, Extremal metrics of zeta function determinants on 4manifolds, Ann. Math., 142(1995)171-212.

[5] M.Struwe, Curvature flows on surfaces, Annali Sc. Norm. Sup. Pisa, Ser. V, $1(2002) 247-274$.

[6] A.Malchiodi, M.Struwe, Q-curvature flow on $S^{4}$, J.Diff. Geom., 73(2006)1-44.

[7] L.Ma, Three remarks on mean field equations, preprint, 2008.

[8] M.C.Hong,L.Ma, Curvature flow to Nirenberg problem, preprint, 2008.

[9] , J.Wei and X.Xu, On conformal deformation of metrics on $S^{n}$, J. Functional Analysis, 157(1998)292-325.

Li Ma, Department of mathematical sciences, Tsinghua University, Beijing 100084, CHINA

E-mail address: Ima@math.tsinghua.edu.cn 